\title{
CÁNCER DE PRÓSTATA COMO HALLAZGO EN RESECCIÓN TRANSURETRAL
}

\author{
Tristán Dellavedova, Rolando Ponzano, Laura Racca, Federico Minuzzi y Mariana Domínguez.
}

FUCDIM (Fundación Urológica Córdoba para la Docencia e Investigación Médica). Córdoba. Argentina.

\begin{abstract}
Resumen.- OBJETIVO: El hallazgo de adenocarcinoma prostático en piezas quirúrgicas sin diagnóstico previo de tumor se presenta en 4 a $15 \%$ de los pacientes. En algunos, existe sospecha previa, en otros, el hallazgo es "incidental". Presentamos 7 casos de cáncer de próstata detectados en 100 pacientes a quienes se les realizó resección transuretral (RTU) bipolar de próstata debido a indicaciones quirúrgicas habituales. El objetivo de este trabajo es describir las características de los pacientes en quienes se encontró cáncer en la resección transuretral y su evolución postoperatoria, analizar la RTU como herramienta diagnóstica y evaluar las opciones de tratamiento.
\end{abstract}

MÉTODO: Entre junio de 2007 y agosto de 2009, se realizaron en FUCDIM 100 RTU bipolares de próstata en pacientes con patología obstructiva. En 7 se detectó adenocarcinoma de próstata. A ningún paciente se le realizó RTU solamente por elevación del antígeno prostático específico (PSA).

RESULTADOS: El promedio de PSA preoperatorio fue $7,6 \mathrm{ng} / \mathrm{ml} / \mathrm{r}=0,72-27 \mathrm{ng} / \mathrm{ml}), 39 \%$ de los pacientes tenían PSA <4 ng/ml; 33 (40\%) pacientes tenían biopsias previas y $36 \%$ de ellos 2 o más biopsias. La tasa global de detección de cáncer de próstata fue de $7 \%$, 3 fueron hallazgos "incidentales" (PSA bajo y tumor de bajo riesgol, 3 con PSA elevado y varias biopsias previas negativas y 1 con PSA bajo y tumor agresivo (Gleason 4+3).

CONCLUSIONES: Los pacientes con cáncer de próstata como hallazgo incidental durante la RTU constituyen un grupo heterogéneo. En pacientes con síntomas obstructivos, PSA elevado y biopsias negativas, la RTU es a la vez diagnóstica y terapéutica. Las alternativas terapéuticas ante el diagnóstico de CaP tras la RTU son variadas y se debe tener en cuenta el grado y estadío del tumor, edad, expectativa de vida y voluntad del paciente. La RTU bipolar permite plantear, en pacientes seleccionados, la posibilidad de ofrecer "active surveillance"(en este grupo es muy útil el PSADT) y de no encontrarse el tumor, facilita el seguimiento de estos pacientes. El tratamiento activo (cirugía o radioterapia) está indicado en pacientes Tl a con expectativa de vida mayor a 10 años, y en la mayoría de pacientes TIb.

Palabras clave: Adenocarcinoma de próstata. Tumor incidental. Resección transuretral bipolar. Resección transuretral diagnóstica.

Summary.- OBJETIVES: Prostate adenocarcinoma is found in surgical samples without prior diagnosis in 4 to $15 \%$ of the patients. In some of them, there is previous 
suspicion but in others this finding is completely incidental. We present 7 cases of prostate cancer detected in 100 patients who underwent bipolar transurethral resection (TUR) of the prostate due to regular indications. The aim of this paper is to describe patient's characteristics, postoperative outcome, analyze TURP as a diagnostic tool and evaluate therapeutic options for prostate cancer (PCa).

METHODS: One hundred patients with bladder outlet obstruction due to benign prostatic hyperplasia (BPH) underwent TURP in FUCDIM between June 2007 and August 2009. In 7 of them, prostate adenocarcinomas were detected. None of the patients underwent TURP only because of increased prostate-specific antigen (PSA).

RESULTS: Mean preoperative PSA was $7.6 \mathrm{ng} / \mathrm{ml} / \mathrm{r}=$ $0.72-27 \mathrm{ng} / \mathrm{mll}$, 39\% of the patients had PSA $<4$ $\mathrm{ng} / \mathrm{ml} ; 33$ (40\%) had undergone previous biopsies and $36 \%$ of them had 2 or more biopsies. Prostate cancer detection global rate was 7\%, 3 cases were incidental findings (low PSA and low-risk tumors), 3 patients had increased PSA and several previous biopsies with negative results and 1 had low PSA and aggressive tumor (Gleason 4+3).

CONCLUSIONS: TURP patients with prostate cancer are a heterogeneous group. TURP can be both diagnostic and therapeutic when facing patients with obstructive symptoms, high PSA and negative prostate biopsies. There are several therapeutic alternatives for TURP patients with cancer, taking into consideration tumor grade and stage, age, life expectancy and will of the patient. Bipolar TUR, in selected patients, allows to offer optional active surveillance (in these patients PSADT is very usefull and if cancer is not found, it eases the follow up of these patients. Active treatment (surgery or radiotherapy) is indicated in Tla patients with life expectancy longer than 10 years, and in the majority of $T 16$ patients.

Keywords: Prostate adenocarcinoma. Incidental tumor. Bipolar transurethral resection. Diagnostic TURP.

\section{INTRODUCCIÓN}

El diagnóstico actual de la mayoría de neoplasias prostáticas se obtiene por biopsias motivadas por elevación del antígeno prostático específico (PSA), cuyo uso está muy difundido (1). Sin embargo, todavía es posible encontrar adenocarcinomas en informes histopatológicos de piezas operatorias de pacientes sin diagnóstico previo. Algunos casos no sorprenden, ya que no pocos pacientes llegan a la cirugía por hiperplasia prostática benigna sintomática con valores elevados de PSA y biopsias preoperato- rias negativas. Otra situación diferente es el hallazgo "incidental" de tumores que probablemente no hubieran sido detectados de no mediar la cirugía ya que no hubieran dado síntomas o elevación del PSA.

Las opciones terapéuticas recomendadas son: vigilancia activa ("active surveillance"), tratamiento local con cirugía o radioterapia externa, y en pacientes con enfermedad avanzada, añosos o que deseen terapias no invasivas se puede plantear bloqueo hormonal.

Presentamos nuestra casuística con los resultados de 7 pacientes en una población de 100 operados a quienes se les detectó adenocarcinoma de próstata al realizárseles resección transuretral (RTU) bipolar de próstata por las indicaciones habituales.

El objetivo de este trabajo es describir las características de los pacientes en quienes se encontró cáncer en la resección transuretral y su evolución postoperatoria, analizar a la RTU como herramienta diagnóstica y evaluar las opciones de tratamiento en estos casos.

\section{MATERIAL Y MÉTODOS}

Se analizaron de manera retrospectiva y descriptiva, 100 casos de RTU bipolar de próstata en pacientes con patología obstructiva, excluyendo aquellos con diagnóstico previo de cáncer de próstata (CaP). Todos los procedimientos fueron realizados en FUCDIM entre junio de 2007 y agosto de 2009 por el mismo profesional y todas las muestras fueron analizadas por el mismo uropatólogo.

Se confeccionó una planilla con los datos de cada paciente antes de la cirugía (nombre, edad, volumen prostático, indicación quirúrgica, PSA, biopsias previas, puntaje internacional de síntomas prostáticos-IPSS y flujometría), datos de las cirugías (tiempo, gramos resecados, horas de internación, resultado histopatológico) y datos postoperatorios (PSA, complicaciones, resultado funcional).

Ante el hallazgo de cáncer de próstata, se estadificó a los pacientes con tomografía computada abdómino-pélvica y centellograma óseo y se realizó seguimiento en todos los casos, independientemente del tratamiento elegido.

\section{RESULTADOS}

Las principales indicaciones del tratamiento quirúrgico fueron prostatismo $(58 \%)$, retención urina- 
TABLA I. CARACTERISTICAS PRE, INTRA Y POSTOPERATORIAS (PROMEDIOS) DE PACIENTES SIN Y CON CÁNCER DE PRÓSTATA EN RTU.

\begin{tabular}{|c|c|c|c|c|c|c|c|c|c|}
\hline Grupo & № & Edad & Gramos & PSA & Biopsia & Gramos & Tiempo \\
preoperatorio & preoperatorio & previa & RTU & quirúrgico & internación & Complicaciones \\
\hline Sin CaP & 93 & 65 & 99,2 & 7,6 & $39 \%$ & 78,6 & 81 & 16 & $18 \%$ \\
\hline Con CaP & 7 & 69 & 74,5 & 6,4 & $57 \%$ & 71,4 & 60 & 16 & - \\
\hline
\end{tabular}

CaP: cáncer de próstata.

ria, infecciones recurrentes, litiasis o hematuria iterativa. A ningún paciente se le indicó la RTU sólo por PSA elevado, sí en algunos casos en que el mismo se asociaba a síntomas prostáticos. En la Tabla I se presentan los resultados de los pacientes con y sin cáncer de próstata.

El grupo con cáncer presentó mayor promedio de edad, menor volumen preoperatorio, PSA levemente menor, alto porcentaje de biopsias preoperatorias negativas, menos gramos resecados, menor tiempo quirúrgico, igual tiempo de internación y no tuvo complicaciones perioperatorias comparado con con el grupo sin CaP.
La tasa global de detección de CaP fue de $7 \%$, siendo de $4 \%$ en los pacientes que no tenían biopsias previas y de $12 \%$ en aquellos que sí la tenían.

No hubo reintervenciones de urgencia ni mortalidad perioperatoria. Los resultados funcionales postoperatorios fueron satisfactorios (objetivados por "score" de síntomas, calidad de vida, flujometría).

El promedio de PSA postoperatorio fue 1 $\mathrm{ng} / \mathrm{ml}$. Del $61 \%$ de PSA preoperatorios $>4 \mathrm{ng} / \mathrm{ml}$, ninguno repitió valores en ese rango tras la cirugía, ni siquiera aquellos con diagnóstico de cáncer.

\section{TABLA II. CARACTERÍSTICAS DE LOS PACIENTES CON HALLAZGO DE CÁNCER EN LA RTU.}

\begin{tabular}{|c|c|c|c|c|c|c|c|}
\hline $\begin{array}{c}\text { Paciente } \\
\text { No }\end{array}$ & Edad & PSA & $\begin{array}{c}\text { Bx } \\
(-)\end{array}$ & Localización tumor & Gleason & $\begin{array}{c}\% \text { tumor en } \\
\text { tejido RTU }\end{array}$ & $\begin{array}{c}\text { ler PSA } \\
\text { postop }\end{array}$ \\
\hline 1 & 58 & 10 & 2 & Periférico & $3+3$ & $<5 \%$ & 0,5 \\
\hline 2 & 69 & 18 & 5 & Periférico bilateral & $3+3$ & $<5 \%$ & $0,1+$ \\
\hline 3 & 63 & 7,7 & 4 & Transicional y periférico & $3+3$ & $<5 \%$ & 0,58 \\
\hline 4 & 70 & 5,4 & 1 & Transic y perifér, "incidental" & $3+3$ & $<5 \%$ & 0,26 \\
\hline 5 & 82 & 0,9 & - & Transicional, "incidental" & $3+3$ & $<5 \%$ & 0,1 \\
\hline 6 & 67 & 1,2 & - & Transicional, "incidental" & $3+3$ & $<5 \%$ & 0,47 \\
\hline 7 & 77 & 2,6 & - & Transicional y periférico & $4+3$ & $90 \%$ & 0,1 * \\
\hline
\end{tabular}

* Recibió bloqueo hormonal tras el diagnóstico

+ Recibió bloqueo hormonal y radioterapia tras el diagnóstico

Bx: biopsia 
Ningún paciente tenía diagnóstico previo de cáncer y en 7 casos se lo diagnosticó mediante el análisis de los fragmentos. En el resto no se halló malignidad, detectándose prostatitis granulomatosa en 2 casos.

El material obtenido en la RTU se almacena por separado en cuatro recipientes, identificados como transicional y periférico derecho e izquierdo, excepto en los raros casos en los que se reseca escasa cantidad de tejido (en todos los casos con cáncer se extrajeron $30 \mathrm{gr}$ o más, y todas las muestras fueron enviadas por separado).

El porcentaje de tumor en el tejido resecado fue menor a $5 \%$ en los casos 1 a 6 lentre 0,1 y $0,5 \%)$, o sea estadío Tla, siendo el paciente no 7 el único caso con gran volumen tumoral $190 \%$ de tejido resecado con cáncer), quien en realidad, como tenía tacto sospechoso, correspondería a estadío T2.

Las características de los 7 pacientes con cáncer se detallan en la Tabla II. Seis de ellos tenían tacto rectal normal. Los estudios de estadificación realizados en 6 pacientes fueron compatibles con tumores localizados. El único caso que no se estadificó fue el paciente más añoso, que tuvo complicaciones debidas a leucemia previamente diagnosticada y falleció a los 8 meses de operado.

Al paciente $\mathrm{n}^{\circ} 4$ se le diagnosticó el CaP al ser operado por carcinoma urotelial de vejiga, que infiltraba pared vesical y próstata. Por este motivo se resecó parte de la glándula, que estaba comprometida por el carcinoma de vejiga (comprobado por inmunohistoquímica), y además, en esos fragmentos se encontró el adenocarcinoma "incidental" de próstata.

Como se mencionó previamente, todos los pacientes, aún aquellos con PSA inicial elevado, tuvieron valores postoperatorios $<1 \mathrm{ng} / \mathrm{ml}$ y sólo dos de ellos recibieron bloqueo hormonal. El tiempo promedio de seguimiento fue de 20 meses (rango $=12$ 29). Los pacientes $n^{\circ} 5$ y 7 fallecieron (el primero fue hallazgo "incidental" y el otro presentaba un tumor extenso), a los 15 y 18 meses de la cirugía respectivamente, por causas no relacionadas a la misma ni al adenocarcinoma de próstata. De los pacientes restantes, 3 permanecen sin tratamiento activo ("active surveillance") con tiempo de duplicación del PSA (PSADT) de 13, 17 y 20 meses respectivamente; el paciente con cáncer urotelial infiltrante recibió quimo-radioterapia y otro paciente radioterapia externa (caso $n^{\circ} 2$ ). En todos los casos se explicó claramente a los pacientes los riesgos y beneficios de cada opción y se consensuó con ellos el tratamiento.
El paciente $\mathrm{n}^{\circ} 7$ tenía tacto rectal sospechoso para cáncer, pero también prostatismo severo (catéter uretral permanente) y comorbilidades importantes. Se decidió realizar RTU para desobstruirlo y a la vez lograr material para biopsia.

\section{DISCUSIÓN}

En el marco de las cirugías por patología prostática obstructiva benigna puede detectarse un adenocarcinoma. En algunos casos, como en la mayor parte de nuestra muestra, se utiliza la RTU en pacientes con obstrucción infravesical sintomática para detectar tumores sospechados por elevación del PSA que no habían podido ser encontrado en biopsias por punción ("RTU diagnóstica"). En otros casos los tumores son hallazgos y probablemente, de no haber requerido ese paciente la cirugía desobstructiva, nunca se los hubiese detectado clínicamente (tumores "incidentales"). La tercera situación presentada es el diagnóstico de una neoplasia con criterios de agresividad (score de Gleason $7,4+3$ ) en un paciente con sospecha de cáncer por tacto rectal y con obstrucción infravesical franca.

La RTU de próstata no es considerada actualmente una herramienta para el diagnóstico de adenocarcinoma de próstata, excepto en casos aislados. La cirugía bipolar es una herramienta nueva, en uso desde hace menos de 10 años, que permite la remoción de tejido prostático con irrigación continua de solución fisiológica, que al ser isotónica con el plasma, evita el riesgo de "síndrome de absorción", la complicación más temida de la RTU monopolar (2). Ni en la literatura consultada ni en nuestra serie comparativa se encontraron diferencias entre RTU mono y bipolar respecto a detección de cáncer $(2,3)$. Sin embargo, al otorgar mayor seguridad en la resección, es posible hacerla en forma más acabada y sin límite de tiempo, permitiendo resecar más tejido y por lo tanto detectar mejor los tumores prostáticos. Una RTU es considerada adecuada cuando se reseca al menos un tercio del volumen de la glándula(4); en nuestra casuística, la media de tamaño prostático preoperatorio fue de $99,2 \mathrm{gr}$ y los gramos resecados 78,6 , o sea que se resecó $78 \%$ de las glándulas en promedio.

En pacientes con PSA elevado o en aumento y biopsias transrectales negativas, la bibliografía consultada sugiere repetir biopsias con inclusión de la zona de transición, luego hacer "biopsias por saturación", de 24 a 48 tomas con anestesia general. Se está estudiando, con resultados promisorios, la resonancia magnética con espectroscopía para detectar áreas sospechosas y poder dirigir nuevas biopsias; y por último, la "RTU diagnóstica"(5). 
Por otro lado, la "National Comprehensive Cancer Network" de EEUU, plantea el siguiente algoritmo: biopsia de 12 tomas, si es negativa repetirla, considerando incluir la zona de transición, si es nuevamente negativa y el paciente es de alto riesgo, considerar la biopsia "por saturación" (6).

La realización de una RTU sólo para detectar cáncer no está aceptada como indicación formal, excepto que los pacientes tengan biopsias negativas y refieran síntomas obstructivos que justifiquen la intervención. Al respecto, van Renterghem estudió pacientes asintomáticos con HPB por medio de urodinamia detectando obstrucción infravesical en la mayoría de ellos, por lo que propuso realizar cirugías diagnósticas con el triple objetivo de aliviar la obstrucción (aún si fuera asintomática), hacer biopsias extensas y disminuir los valores de PSA (7).

A pesar de que los argumentos científicos que avalan la "RTU diagnóstica" son escasos, las evidencias a su favor van tomando mayor importancia. Entre estas se pueden mencionar:

- Pacientes asintomáticos con PSA elevado que pueden tener obstrucciones significativas y beneficiarse con la cirugía $(7,8)$. Valores altos de PSA se asocian a riesgo de retención urinaria (9).

- Pacientes con PSA en aumento que pueden tener tumor en la zona transicional, la cual es mejor biopsiada con RTU que con punción transrectal $(8,10)$.

- La RTU tiene baja tasa de complicaciones, que pueden reducirse aún más haciendo resecciones limitadas que eviten la resección apical y la perforación capsular(8)

- Las neoplasias de la zona transicional son generalmente de bajo grado y con menor tendencia a comprometer la cápsula y metastatizar, por lo que hay buena oportunidad de curación, aún con valores de PSA $>20 \mathrm{ng} / \mathrm{ml}(8,11)$.

- El PSA desciende a valores normales en la mayoría de los pacientes (4).

- La próstata remanente es más fácil y completamente biopsiada en biopsias subsiguientes, aumentando la detección de cáncer en rebiopsias post RTU (4).

- Por último, la prostatectomía radical luego de RTU presenta algunas dificultades técnicas, pero en manos experimentadas tiene excelentes resultados oncológicos y funcionales (8).
Los detractores de esta técnica refieren que tras una segunda biopsia prostática negativa, la tasa de detección de cáncer es muy similar con una tercera biopsia (10\% detección), "RTU diagnóstica" (9$12 \%$ detección), o con un seguimiento cuidadoso durante 7 años y biopsia ante elevación del PSA $11 \%$ detección) (8). Paolo Puppo propone como alternativa para casos con al menos dos biopsias negativas, realizar RTU y biopsia transrectal en el mismo acto. Sobre 43 pacientes, detectó cáncer en 8 de ellos $(18,6 \%$ de detección), 6 con RTU $(75 \%)$, uno con biopsia $(12,5 \%)$ y el último en ambas, RTU y biopsia (12). Una publicación reciente de Ploussard muestra resultados opuestos, ya que incluyó 113 pacientes asintomáticos con al menos 2 biopsias negativas de 21 tomas, a quienes se les realizó otra biopsia de 21 muestras e inmediatamente RTU. La tasa de detección global fue de $23,9 \%$, siendo $63 \%$ sólo por biopsia, $22 \%$ sólo por RTU y $15 \%$ en ambas (4).

La detección de adenocarcinoma en pacientes operados por hiperplasia oscila ente 4 y $15 \%$ en la literatura $(13,14,15)$. Según la clasificación de la Unión Internacional Contra el Cáncer (UICC) del año 2002, se establece una diferencia entre Tla (hallazgo de tumor incidental en $5 \%$ o menos del tejido resecado) y T1b (hallazgo de tumor incidental en más del $5 \%$ del tejido resecado) (16). Esto se basa en el riesgo de progresión, que es sólo de $5 \%$ a los 5 años (aunque el $50 \%$ progresa entre 10 y 13 años) para Tla; en cambio, la mayoría de los casos de Tlb progresan a 5 años(16). Esto permite clasificar, por su agresividad potencial, como tumor "insignificante" al primero y tumor "clínicamente significativo" al segundo (17).

El tratamiento con prostatectomía radical para estos dos estadíos tiene riesgo de no poder encontrar tumor (pTO) en 4 a $21 \%$ de los casos. Por el mencionado riesgo de progresión a largo plazo es que a los pacientes jóvenes Tl a con expectativa de vida mayor a 15 años se les ofrece la cirugía. Según las recomendaciones de la Sociedad Europea de Urología año 2010 ante lesiones T1b, la prostatectomía radical es el "standard" de tratamiento, en particular ante pacientes con expectativa de vida mayor a 10 años, agrupando esta última categoría con la de tumor palpable localizado en un lóbulo prostático (T2a-T2b); al contrario, el "standard" para lesiones Tla es "active surveillance" (16).

Monda postula que el uso rutinario de PSA no es útil para disminuir la prevalencia de tumores Tla, ya que no diferencia estos tumores de hiperplasia, pero sí puede disminuir la prevalencia de lesiones $T 1 b$, que generalmente provocan elevación del PSA (18). En nuestra casuística no hubo casos T1b. 
Se cree que muchos de los tumores de pacientes con biopsias previas negativas están localizados en la zona de transición, especialmente en la parte anterior. Estos tumores son de bajo grado, poco agresivos y con altas probabilidades de curación $(8,11)$. Otros autores, en cambio, sostienen que el comportamiento de estas lesiones puede ser agresivo y que frecuentemente se asocian con tumores satélites en la zona periférica, de mayor tamaño y mayor grado, como se ha comprobado en piezas de prostatectomía $(4,19)$.

Algunos autores informan una incidencia real muy baja de tumores transicionales puros $(0,6 \%)$ y consideran que no es de utilidad realizar de tomas biopsias en esta área (20); por el contrario, Ploussard reporta $19 \%$ de tumores de la zona de transición exclusivamente(4). De nuestros casos sólo dos fueron transicionales puros, ambos "incidentales" $y$ con PSA bajo $(0,9$ y $1,3 \mathrm{ng} / \mathrm{ml})$.

La realización de biopsias prostáticas ante el hallazgo de cáncer tras RTU puede ser útil para detectar tumor residual, tumor en zona periférica no diagnosticado o para lograr una mejor valoración del grado tumoral (16). De nuestros pacientes, ninguno fue rebiopsiado, ya que sólo dos tenían tumor transicional puro, uno de ellos falleció por otras causas y el restante tiene tiempo de duplicación del PSA (PSA doubling time-PSADT) de 17 meses.

En nuestra serie, los tres tumores catalogados como "incidentales" eran Gleason 6 y fueron hallados en 1, 2 y 3 fragmentos (menos de 1\% del tejido) respectivamente. Habitualmente estos pacientes no requieren tratamiento. De estos 3 pacientes, 2 no tenían PSA elevado, eran Tla y no recibieron tratamiento, el tercero tenía un tumor vesical que infiltraba la próstata, lo que podría explicar el PSA de $5,4 \mathrm{ng} / \mathrm{dl} \mathrm{y} \mathrm{la} \mathrm{indicación} \mathrm{de} \mathrm{biopsia} \mathrm{previa} \mathrm{negativa.}$ En este caso, se encontró mucho volumen de próstata comprometido por el tumor urotelial y apenas 3 fragmentos de la RTU (menor a 5\%) con adenocarcinoma. Probablemente, de no haber existido el tumor de vejiga, no se hubieran elevado los valores de PSA ni el paciente hubiera sido biopsiado.

La tercera situación en nuestra serie era especial: se trataba de un paciente añoso con mucha comorbilidad, PSA: $2,61 \mathrm{ng} / \mathrm{ml}$, tacto rectal francamente patológico, próstata de gran tamaño y marcada sintomatología que no respondía a la medicación, por lo que requería el uso de sonda permanente. Esta situación nos obligaba a tomar una decisión: indicar biopsia por punción, con la consiguiente cascada de eventos que siguen al diagnóstico, y aún restaba resolver su cuadro clínico, o hacer a la vez un gesto diagnóstico y terapéutico, realizando la RTU para aliviar la obstrucción, conseguir la biopsia prostática y llevar el PSA a niveles más bajos. Su expectativa de vida era limitada y de hecho, falleció 15 meses después por un evento cardiovascular sin relación con su patología urológica, por lo que nos pareció que el manejo oncológico pasaba a segundo plano. Tras la cirugía, los estudios informaron tumor localizado y el paciente tuvo muy buenos resultados funcionales.

\section{CONCLUSIONES}

Los pacientes con hallazgo de CaP en RTU constituyen un grupo heterogéneo ya que incluyen pacientes con alto índice de sospecha y otros en quienes se encuentran tumores "incidentales". La RTU como herramienta diagnóstica es útil en casos seleccionados, ante pacientes con síntomas obstructivos, PSA elevado y biopsias prostáticas negativas repetidas. Muchos albergan su tumor en la zona transicional, aunque no exclusivamente. Tiene la ventaja de desobstruir al paciente a la vez que obtiene buen material para análisis histopatológico y no debe realizarse sólo por aumento del PSA en ausencia de síntomas prostáticos. El cáncer de próstata tras RTU bipolar plantea diferentes situaciones en cuanto al manejo de la neoplasia, en las que intervienen el estadio y grado del tumor, su volumen, la edad, expectativa de vida y voluntad del paciente. EI PSADT es un buen marcador de actividad tumoral en pacientes en "active surveillance" y puede identificar pacientes que requieren tratamiento activo.

La detección de neoplasia prostática por RTU permite plantear, en pacientes seleccionados, la posibilidad de "active surveillance" y si se decide hacer tratamiento, se puede optar por radioterapia externa, prostatectomía radical o bloqueo androgénico. En caso de no encontrarse tumor, la RTU facilita el seguimiento de estos pacientes aliviando los síntomas, disminuyendo el PSA y mejorando el rendimiento de biopsias posteriores, si fueran necesarias.

\section{BIBLIOGRAFÍA y LECTURAS RECOMENDADAS ( ${ }^{*}$ lectura de interés $y^{* *}$ lectura fundamental)}

1. Carroll P, Albertsen PC, Greene K, Babaian RJ, Ballentine Carter H, Gann PH, et al. Prostate-Specific Antigen: Best Practice Statement. Am Urol Association. 2009 Update.

2. Michielsen DPJ, Debacker T, De Boe V. Bipolar transurethral resection in saline-an alternative surgical treatment for bladder outlet obstruction? J Urol, 2007; 178(5): 2035-9. 
3. Minuzzi Federico. RTU Monopolar y bipolar de próstata. Punto-Contrapunto. Cirugía de la HPB de hasta 80 gramos, Congreso Argentino de Urología, 6-8 de octubre, 2009, Buenos Aires, Argentina.

**4. Ploussard G, Dubosq F, Boublil V, Allory Y, de la Taille A, Vordos MD, et al. Extensive biopsies and transuretral prostate resection in men with previous negative biopsies and high or increasing prostate specific antigen. J Urol, 2009; 182(4): 1342-9.

*5. Bratt $\mathrm{O}$. The difficult case in prostate cancer diagnosis-When is a "diagnostic TURP" indicated? Eur Urol 2006; 49: 769-771.

6. Kawachi M, Babaian RJ, Bahnson RR, Barry M, Busby JE, Carrol PR, et al. NCCN Clinical Practice Guidelines in Oncology. Prostate Cancer Early Detection, 2010.

*7. van Renterghem K, Van Koeveringe G, Achten $\mathrm{R}$, van Kerrebroeck P. Prospective study of the role of transurethral resection of the prostate in patients with an elevated prostate-specific antigen level, minor lower urinary tract symptoms, and proven bladder outlet obstruction. Eur Urol, 2008; 54(6): 1385-92.

**8. Puppo P. Repeated negative prostate biopsies with persistently elevated or rising PSA: a modern urologic dilema. Eur Urol, 2007; 52: 639-41.

9. Margerber MJ, Andersen JT, Nickel JC, Malice MP, Gabriel M, Pappas F et al. Prostate volume and serum prostate-specific antigen as predictors of acute urinary retention. Combined experience from three multinational placebo-controlled trials. Eur Urol, 2000; 38: 563-8.

10. Lin CC. Re: Extensive biopsies and transurethral prostate resection in men with previous negative biopsies and high or increasing prostate specific antigen. J Urol, 2009; 182(4): 1342-9.

11. Steuber T, Karakiewicz PI, Augustin H, Erbersdobler A, Lange I, Haese A, et al. Transition zone cancers undermine the predictive accuracy of Partin table stage predictions. J Urol, 2005; 173: $737-41$.
*12. Puppo P, Introini C, Calvi P, Naselli A. Role of transurethral resection of the prostate and biopsy of the peripheral zone in the same session after repeated negative biopsies in the diagnosis of prostate cancer. Eur Urol, 2006; 49: 873-8.

13. Jones JS, Follis HW, Johnson JR. Probability of finding T1a and T1b (incidental) prostate cancer during TURP has decreased in the PSA era. Prostate Cancer P D, 2009; 12(1): 57-60.

14. Merrill RM, Wiggins CL. Incidental detection of population-based prostate cancer incidence rates through transurethral resection of the prostate. Urol Oncol, 2002; 7(5): 213-9.

15. Martino P, Palazzo S, Battaglia M, Lucarelli G, Selvaggi FP. Incidental prostatic cancer: repeat TURP or biopsy? Urol Int 2004; 73(3): 193-7.

16. Heidendreich A, Aus G, Abbou CC, Bolla M, Joniau S, Matveev V, et al. Guidelines on Prostate Cancer, p. 33. Eur A Urol. Guidelines, 2009 edition.

17. Epstein JI. Can insignificant prostate cancer be predicted preoperatively in men with stage $\mathrm{T} 1 \mathrm{di}-$ sease? Semin Urol Oncol, 1996; 14(3): 165-73.

18. Monda JM, Barry MJ, Oesterling JE. Prostate specific antigen cannot distinguish stage T1a (A1) prostate cancer from benign prostatic hyperplasia. J Urol, 1994; 151(5): 1291-5.

19. Radhakrishnan S, Dorkin TJ, Sheikh N, Greene DR. Role of transition zone sampling by TURP in patients with raised PSA and multiple negative transrectal ultrasound-guided prostatic biopsies. Pr Cancer and P D 2004; 7: 338-42.

20. Pelzer AE, Bektic J, Berger AP, Halpern EJ, Koppelstatter F, Klauser A, et al. Are Transition Zone Biopsies Still Necessary to Improve Prostate Cancer Detection? Results from the Tyrol Screening Project. Eur Urol, 2005; 48: 916-21.

21. Sakr WA; Billis A; Ekman P; Wilt T; Bostwick DG. Epidemiology of High-Grade Prostatic Intraepithelial Neoplasia. Scand J of Urol Nephr, 2000; 34 S1 (205): 11-8. 